\title{
Diagnostic accuracy value of intraoperative frozen section for ovarian neoplasms
}

\section{Farinaz Shahmoradi}

Shaheed Beheshti University of Medical Sciences

Maliheh Arab ( $\nabla$ drmarab@yahoo.com )

Shaheed Beheshti University of Medical Sciences

\section{Research}

Keywords: Frozen Section, Ovarian Neoplasms, Ovary, Diagnostic Test, Sensitivity

Posted Date: March 25th, 2020

DOI: https://doi.org/10.21203/rs.3.rs-19106/v1

License: (c) (i) This work is licensed under a Creative Commons Attribution 4.0 International License. Read Full License

Version of Record: A version of this preprint was published at Indian Journal of Gynecologic Oncology on June 27th, 2020. See the published version at https://doi.org/10.1007/s40944-020-00425-4. 


\section{Abstract}

Background Accuracy of intraoperative frozen section diagnosis is extremely important in the evaluation of ovarian tumors so that suitable surgical procedures can be chosen.

Methods This retrospective diagnostic accuracy study was conducted in the Department of Obstetrics and Gynecology, Emam Hossein Hospital. The case records of patients with ovarian mass who underwent surgery and intraoperatively frozen section assessments between September 2006 and December 2014 were analyzed. Demographic and clinical data, operative details, frozen section and final histopathology Examination (HPE) were reviewed from the medical records.

Results one hundred and ninety three patients with ovarian masses undergoing surgery and frozen section were included. The frozen section diagnoses were benign in $155(80.31 \%)$, borderline in 10 (5.18\%), and malignant in 28 (14.51\%), whereas the final diagnosis was benign in 154 (79.80\%), borderline in 9 (4.66\%), and malignant in 30 (15.54\%). The overall accuracy of intra-operative frozen section diagnosis was $98.9 \%$. The sensitivity for frozen section diagnosis was $100 \%$ for benign, $89 \%$ for borderline and $93.3 \%$ for malignant category, whereas the specificity was $97 \%, 99 \%$, and $100 \%$, respectively. There were 3 cases with discordance between the frozen section diagnoses and the final diagnoses, all of which were under diagnosed by frozen section.

Conclusion Frozen section was found to be accurate and useful in the intraoperative assessment of patients with ovarian neoplasm. The results may help to determine the type and extent of surgery.

\section{Introduction}

Ovarian neoplasms are a significant cause of female morbidity and mortality (1). Ovarian malignancy accounts for nearly $25 \%$ of gynécological cancers and $50 \%$ of all deaths from female genital cancers (2). It is one of the top 10 cancers in the US with an estimated 22,530 new cases and 13980 deaths in 2019 (3).

Ovarian tumor is frequently diagnosed in females of all ages, including reproductive age groups. Such women have about $10 \%$ need for surgery and about 13 to $21 \%$ were diagnosed with malignant ovarian tumor in those who underwent surgery (4). Malignancy diagnosis is generally simple, but in some cases it may be hard to differentiate between the ovarian origin of the malignant tumor or metastases from other areas $(5,6)$. In order to determine the extent of the procedure, it is essential to know the intraoperative condition of the tumor. Furthermore, it is important to distinguish between malignant and benign pathology before and during surgery to ensure optimal management of the patient with an adnexal mass. In case of doubt about the condition of malignancy prior to surgery, or in patients in whom the existence of malignancy prior to surgery is unsure, assessment of the frozen section may provide additional data during surgery (7). Since 1891, this method has been known but studies on its diagnostic accuracy were performed just two decades ago (8). Frozen section is commonly used for intraoperative assessment of patients with ovarian tumors in order to provide instructions for effective surgical administration. The 
intraoperative records of frozen tissue taken during surgery are appealing, because the diagnosis can be provided intraoperatively. Such a diagnosis can notify the surgeon not only of the malignant nature of the ovarian lesion, but also of the potential for metastasis (9). Diagnostic problems can arise especially in mucinous and borderline tumors due to technical limitations (2). In order to obtain accurate results and to reduce the number of deferred cases, effective interaction between clinicians and pathologists is needed (10).

Preoperative imaging and tumor markers have only limited value in differentiating between benign, borderline and malignant categories (11). Intraoperative frozen section examination can enable the gyneocology team to decide on the type of surgery to be performed in patients with ovarian tumors, especially when there is a concern about fertility. It provides for a single optimum operating staging technique where indicated and, if not necessary, also for a non-staging procedure. Therefore, the current study was aimed at measuring the diagnostic accuracy of the intraoperative frozen section as a piece of evidence to be used by surgeons to determine the surgical course of benign and malignant tumors.

\section{Materials And Methods}

\section{Study design and patients}

This retrospective diagnostic accuracy study was conducted in the Department of Obstetrics and Gynecology, Emam Hossein Hospital, ShahidBeheshti University of Medical Sciences, after being approved by the ethics committee. The case records of patients with ovarian mass who underwent surgery and intraoperatively frozen section assessments between September 2006 and December 2014 were analyzed in this study.

\section{Procedures}

Demographic, operative details, frozen section and final histopathology Examination (HPE) were reviewed from the medical records. The request for an intraoperative frozen section was based on an individual attending gynecologist following a preoperative assessment, including clinical history and examination, biomarkers and radiological imaging.

In our hospital, we have frozen section service for suspected ovarian cancer cases. The GynOncology surgical team informs the laboratory in advance of the need for frozen section. The specimen, once removed, is transported by a hospital porter direct to the laboratory and handed over to the laboratory staff. The pathologist assigned for frozen section inspects the specimen and, after describing it, takes representative pieces of tissue for frozen section analysis. These are then processed and, after hand staining, are given to the duty pathologist for reporting. The average time taken for the entire procedure (after specimen being received) was approximately 15 minutes. The frozen section diagnosis was deferred when there were some suspicious pathologic features of borderline or malignant tumor which were considered not definitely diagnostic. After the frozen section diagnosis was reported, the ovarian 
specimens were fixed in $10 \%$ formalin overnight and sampled for routine histologic sections. The histologic diagnosis of ovarian lesions was based on the WHO Classification (12).

Frozen section diagnoses were compared to the final histologic diagnoses in each case, which were considered as the gold standard. The diagnoses were categorized as benign, borderline and malignant. Agreement of both frozen section and final diagnoses was considered when both diagnoses were identical or were within the same subgroup of malignancy (i.e. epithelial, sex cord-stromal, germ cell, and metastatic).

\section{Statistical analysis}

All data analyzed using STATA software Version 15 (STATA Corp, College Station, Texas). Descriptive study was used to summarize the demographic characteristics of the women. Diagnostic parameters including accuracy, sensitivity, specificity, positive and negative predictive values of frozen section for the diagnosis were calculated. The details of all cases with discordant diagnosis between frozen section and final HPE were reviewed.

\section{Results}

A total of 193 cases of ovarian tumors were examined by intraoperative frozen section and final HPE in this study. The mean age was $45 \pm 6$ years and $46.7 \%$ of them were postmenopausal. The majority of patients of ovarian cancers $(70 \%)$ were preoperatively categorized as stage I disease.

Three cases fell in the discordant category, while 190 cases showed same results on frozen section as well as on permanent section diagnosis. Out of these cases of ovarian tumor, 155 were benign, 10 borderline, and 28 malignant tumors after intraoperative frozen section evaluation (Table 1). Among 155 cases of benign tumors reported by intraoperative frozen section, 154 were confirmed in the final HPE. One case confirmed borderline mucinous tumor in the final HPE. Furthermore, among 10 cases of borderline tumors, 2 cases were underreported in the intraoperative frozen section evaluation, and confirmed to be malignant endometrioid and mucinous lesions at the final HPE. In comparison, all 38 cases of malignant tumors during frozen section evaluation were confirmed malignant at the final HPE (Table 1). The overall accuracy of intraoperative frozen section diagnosis was $98.9 \%$. The sensitivity of frozen section diagnosis was $100 \%$ for benign, $89 \%$ for borderline and $93.3 \%$ for malignant category, whereas the specificity was $97 \%, 99 \%$, and $100 \%$, respectively. The positive predictive value was $99 \%$ for benign, $80 \%$ for borderline, and $100 \%$ for malignant group, and the negative predictive values were $100 \%$, for all categories (Table 2). Again, there were 3 cases with discordance between the frozen section diagnoses and the final HPE, all of which were under-diagnosed by frozen. Two cases were under diagnosed as benign and borderline mucinous tumor by frozen section, while one case was diagnosed as borderline epithelial tumor by frozen section and ultimately malignant endometrioid by final HPE (Table 3). 
Table 1

Comparison between Frozen Section diagnosis and final histopathology Examination $(n=193)$.

\begin{tabular}{|lllll|}
\hline Frozen Section & \multicolumn{4}{l|}{ Final Histopathology Examination } \\
\cline { 2 - 5 } & Benign & Borderline & Malignant & Total \\
\hline Benign & 154 & 1 & 0 & 155 \\
\hline Borderline & 0 & 8 & 2 & 10 \\
\hline Malignant & 0 & 0 & 28 & 28 \\
\hline Total & 154 & 9 & 30 & 193 \\
\hline
\end{tabular}

Table 2

Diagnostic Value of Intraoperative Frozen Section reporting as Benign, Borderline and Malignant Lesions in compared to final histopathology Examination

\begin{tabular}{|llll|}
\hline Diagnostic Value & Benign & Borderline & Malignant \\
\hline Sensitivity \% & 100 & 89 & 93.3 \\
\hline Specificity \% & 97 & 99 & 100 \\
\hline PPV \% & 99 & 80 & 100 \\
\hline NPV \% & 100 & 100 & 100 \\
\hline \multicolumn{4}{l}{ PPV: Positive Predictive Value, NPV: Negative Predictive Value } \\
\hline
\end{tabular}


Table 3

Pathologic and clinical characteristics of three cases with discordant diagnosis between frozen section and final histopathology Examination

\begin{tabular}{|llll|}
\hline Characteristics & Case 1 & Case 2 & Case 3 \\
\hline Age (year) & 43 & 33 & 60 \\
\hline $\begin{array}{l}\text { Size-sonography } \\
(\mathrm{mm})\end{array}$ & $53 \times 42$ & $84 \times 48$ & 150 \\
\hline $\begin{array}{l}\text { Size-Macroscopy } \\
(\mathrm{mm})\end{array}$ & $60 \times 40 \times 20$ & $75 \times 55 \times 25$ & 170 \\
\hline Frozen Section & $\begin{array}{l}\text { Benign mucinous } \\
\text { tumor }\end{array}$ & $\begin{array}{l}\text { Borderline epithelial } \\
\text { tumor }\end{array}$ & $\begin{array}{l}\text { Borderline mucinous } \\
\text { tumor }\end{array}$ \\
\hline Final HPE & $\begin{array}{l}\text { Borderline mucinous } \\
\text { tumor }\end{array}$ & $\begin{array}{l}\text { Malignant endometrioid } \\
\text { tumor }\end{array}$ & $\begin{array}{l}\text { Malignant mucinous } \\
\text { tumor }\end{array}$ \\
\hline HPE: histopathology & Examination; mm: millimeter & \\
\hline
\end{tabular}

\section{Discussion}

We performed a diagnostic accuracy study on the accuracy of frozen section diagnosis in the assessment of the adnexal mass. Final histopathologic diagnosis used as the gold standard. The diagnostic accuracy indices were estimated for frozen section. In the present study, the overall accuracy of frozen section diagnosis of ovarian masses was $98.9 \%$.

The high precision of the frozen section helps gyn-oncologists make reasonable intraoperative decisions and thus in the vast majority of benign cases prevent unnecessary morbidity of the procedure. The accuracy of the frozen section for ovarian tumors varies across different institutions. Subbian et al. found an overall accuracy of $84.2 \%$ in a study involving 135 cases of ovarian tumors (13). Several studies have shown that frozen section diagnosis of ovarian tumors is a robust technique with overall accuracy ranging from $71.9-97 \%(10,14)$. In this study, the overall accuracy of the frozen section diagnosis by intraoperative ovarian mass was $98.9 \%$, which is consistent with previous reports (90-97\%) $(1,6,15-18)$.

The sensitivity of frozen section to a benign tumor from our observation was $100 \%$. Previous studies also reported a high sensitivity ranging from $92.8 \%-100 \%(10,11,19-21)$. In the present study, one borderline tumor was diagnosed as benign by frozen section and that was mucinous tumor. Five borderline tumors, diagnosed as benign on the frozen section, were reported in a tertiary center experience by (21). Several explanations have been given for the relative inaccuracy of the frozen section in the diagnosis of borderline tumors. There may be just a few focal points of frank malignancy in a large borderline tumor which may require a large number of frozen section samples for diagnosis. This is quite labor intensive and typically beyond most laboratory abilities (22). It is remarkable that for borderline 
tumor diagnosis, most studies have reported low sensitivity values. Given the low accuracy rate for borderline ovarian tumors, care and attention are required to develop this field $(10,11,13,14,18)$. Md Arshad et al. observed that the borderline tumor sensitivity was $76.2 \%$ (21). Subbian et al., on the other hand, recorded the lowest borderline tumor sensitivity (31.2\%), especially in the mucinous classification (13). In a review of 60 patients of ovarian tumors by (11), frozen section had low sensitivity (75\%) and PPV (50\%) for borderline tumors. In a further retrospective study of 282 cases, the sensitivity of the frozen section to borderline tumors was $95.8 \%$ and the specificity $97.6 \%$ (23). For the borderline category, they found the lowest positive predictive value (79.3\%), all with epithelium of mucinous type. Our results showed $89 \%$ for sensitivity and $80 \%$ for positive predictive value, which were in range of similar studies. In the present study, three discordant cases were identified during diagnosis; two cases were incorrectly diagnosed as borderline epithelial tumor and borderline mucinous tumor using a frozen section. The first was a malignant endometrioid tumor, and the second was a malignant mucinous tumor of Final HPE. Mucinous tumors are usually large with a heterogeneous component including benign, borderline and malignant constituents in the same tumor, making diagnosis difficult $(18,21,24)$. In addition, Gultekin et al. suggested that the size of the tumor, the presence of a solid component and preoperative CA 125 levels may have an impact on diagnosis (25). However, Palakkan et al. found that there was no statistically significant association between raised CA 125 level and ovarian malignancy (11).

Most studies typically reported a sensitivity of $71-100 \%$ for the detection of malignancies by frozen section, and a specificity ranging from $96-100 \%(11,19,21,23,26,27)$. In our study, the sensitivity of malignant ovarian tumor detection was $93.3 \%$ and the specificity was $100 \%$, reflecting that the frozen section examination was highly sensitive and specific to ovarian malignancy. It is very important to correctly detect malignant ovarian tumors, as it determines the type of surgery that should be performed. A study by (28) indicated that, if the pathologist manager had under-specialty training in gynecological pathology, prevention of sampling errors and misinterpretation, along with effective communication could be ensured. If a frozen section is required during surgery, the Gyne-oncology team should be able to communicate preoperatively with the anesthetist and pathologist. A detailed clinical history and intraoperative observations should be communicated to the pathologist as they may help the pathologist to get the correct diagnosis, especially in problematic cases. Despite advances in histological and molecular techniques, frozen section evidently remains an effective tool to be considered during operational procedures.

Frozen section procedure could reduce the possibilities of doing incomplete surgery for benign tumors (oophorectomy or cystectomy) or radical surgery for malignant conditions, in a majority of patients. Frozen section is sometimes referred to as intraoperative consultation, as its practice usually involves a liaison between the surgeon and gynecological pathologist, rather than the mere provision of a histological diagnosis. Accuracy of frozen section depends on a number of factors such as the number of specimens processed, number of slices of specimen analyzed and nature of tissue. Of note is that the report of benign histology on frozen section is only a report of that percentage of tissue analyzed.18 Therefore, intraoperative frozen section is necessary, both as a quick histologic diagnosis and as a guide for the surgeons in planning an appropriate management. 
Besides the nature of the tumor affecting frozen section examination, frozen artifact may also cause misinterpretation of frozen section. The ovarian tumor that was sent to the laboratory is preferably frozen in liquid nitrogen. It is important that the tissue be frozen as soon as possible to prevent ice crystal formation resulting in artifact and poor morphological preservation of the tissue, rendering poor slide quality and difficulty to obtain a diagnosis. Another factor that may contribute to misinterpretation is the presence of necrosis. A tumor that has become necrotic following torsion might have limited tissue available for frozen section evaluation.

The strength of this study was the fact that all slides from both frozen section and final HPE for all the unmatched cases were traced and reevaluated by the pathologist to determine the possible reason for any difference in the result or interpretation. However, several limitations were identified in this study. Firstly, the sample size was limited. Secondly, we did not have all the data on the progress of the patients after the intraoperative frozen section and surgery. Thirdly, the cost effectiveness of intraoperative frozen section was not assessed in our study. Finally, if another blinded pathologist was recruited to determine the reasons for the mismatch, this might decrease our study bias.

\section{Conclusion}

In conclusion, we found a high sensitivity and specificity of the frozen section for the rapid diagnosis of ovarian tumors and for the determination of their malignant potential. Therefore, it should always be used when the preoperative diagnosis is not specific to the extent of the surgical resection. However, under-diagnosis may arise in borderline and malignant tumors, in particular those of mucinous histology, which may be minimized by increased sampling in the frozen section.

\section{Declarations}

Ethical Approval:

The study was approved by the Ethic Committee of Shahid Beheshti University of Medical Sciences (IR.SBMU.MSP.REC.1395.23).

Consent for publication

All of the participants signed a written informed consent prior to participating in this study.

Availability of data and materials

The data will be available for editors in case of reasonable requests.

Competing Interests:

No authors have conflict of interest related with this

Funding:

There is no funding for this study.

Author Contributions:

MA was the principal investigator of this study. FS contributed to the development of the project and performed the statistical analyses. MA and FS contributed development of the project and gathering the 
data. MA and FS contributed the writing the manuscript. All authors contributed to the drafting of this paper and approved the final manuscript.

Acknowledgment:

We would like to thank the staff of the laboratory of Shahid Beheshti University of Medical Sciences who performed the frozen section and final HPE assessments. This article has been extracted from the thesis written by Mrs. Farinaz Shahmoradi in school of medicine Shahid Beheshti University of medical sciences. (Registeration No: 256M)

\section{References}

1. Maheshwari A, Gupta S, Kane S, Kulkarni Y, Goyal BK, Tongaonkar HB. Accuracy of intraoperative frozen section in the diagnosis of ovarian neoplasms: experience at a tertiary oncology center. World journal of surgical oncology. 2006;4:12.

2. Mills AM, Shanes ED. Mucinous Ovarian Tumors. Surgical pathology clinics. 2019;12(2):565-85.

3. Siegel RL, Miller KD, Jemal A. Cancer statistics, 2019. CA: a cancer journal for clinicians. 2019;69(1):7-34.

4. Graham L. ACOG releases guidelines on management of adnexal masses. American family physician. 2008;77(9):1320-3.

5. Avesi L, Kayani N, Hassan S, Shahid R. Diagnostic accuracy of intraoperative consultation of ovarian neoplasms. Asian Journal of Medical Sciences. 2014;6.

6. Stewart CJ, Brennan BA, Hammond IG, Leung YC, McCartney AJ. Intraoperative assessment of ovarian tumors: a 5-year review with assessment of discrepant diagnostic cases. International journal of gynecological pathology : official journal of the International Society of Gynecological Pathologists. 2006;25(3):216-22.

7. Geomini P, Bremer G, Kruitwagen R, Mol BW. Diagnostic accuracy of frozen section diagnosis of the adnexal mass: a metaanalysis. Gynecologic oncology. 2005;96(1):1-9.

8. Rotterdam H. Frozen section diagnosis of serous epithelial tumors of the ovary. Am J Diagn Gynecol Obset. 1979;1:89-94.

9. Cross PA, Naik R, Patel A, Nayar AG, Hemming JD, Williamson SL, et al. Intra-operative frozen section analysis for suspected early-stage ovarian cancer: 11 years of Gateshead Cancer Centre experience. BJOG : an international journal of obstetrics and gynaecology. 2012;119(2):194-201.

10. Ilvan S, Ramazanoglu R, Ulker Akyildiz E, Calay Z, Bese T, Oruc N. The accuracy of frozen section (intraoperative consultation) in the diagnosis of ovarian masses. Gynecologic oncology. 2005;97(2):395-9.

11. Palakkan S, Augestine T, Valsan MK, Vahab KPA, Nair LK. Role of Frozen Section in Surgical Management of Ovarian Neoplasm. Gynecology and minimally invasive therapy. 2020;9(1):13-7.

12. Scully R, Bonfiglio T, Kurman R, Silverberg S, Wilkinson E. World Health Organization International Histological Classification of Tumours. Histological Typing of Female Genital Tract Tumours. New 
York, NY: Springer-Verlag. 1994;19(9):4.

13. Subbian A, Devi UK, Bafna UD. Accuracy rate of frozen section studies in ovarian cancers: a regional cancer institute experience. Indian journal of cancer. 2013;50(4):302-5.

14. Tempfer CB, Polterauer S, Bentz EK, Reinthaller A, Hefler LA. Accuracy of intraoperative frozen section analysis in borderline tumors of the ovary: a retrospective analysis of 96 cases and review of the literature. Gynecologic oncology. 2007;107(2):248-52.

15. Gol M, Baloglu A, Yigit S, Dogan M, Aydin C, Yensel U. Accuracy of frozen section diagnosis in ovarian tumors: Is there a change in the course of time? International journal of gynecological cancer : official journal of the International Gynecological Cancer Society. 2003;13(5):593-7.

16. Boriboonhirunsarn D, Sermboon A. Accuracy of frozen section in the diagnosis of malignant ovarian tumor. The journal of obstetrics and gynaecology research. 2004;30(5):394-9.

17. Tangjitgamol S, Jesadapatrakul S, Manusirivithaya S, Sheanakul C. Accuracy of frozen section in diagnosis of ovarian mass. International Journal of Gynecologic Cancer. 2004;14(2):212-9.

18. Suprasert P, Khunamornpong S, Phusong A, Settakorn J, Siriaungkul S. Accuracy of intra-operative frozen sections in the diagnosis of ovarian masses. Asian Pacific journal of cancer prevention : APJCP. 2008;9(4):737-40.

19. Mohammed AB, Ahuja $V$, Farghaly $H$. Role of frozen section in the intraoperative management of ovarian masses. Middle East Fertility Society Journal. 2014;20.

20. Hashmi AA, Naz S, Edhi MM, Faridi N, Hussain SD, Mumtaz S, et al. Accuracy of intraoperative frozen section for the evaluation of ovarian neoplasms: an institutional experience. World journal of surgical oncology. 2016;14:91-.

21. Md Arshad NZ, Ng BK, Md Paiman NA, Abdullah Mahdy Z, Mohd Noor R. Intra-Operative Frozen Sections for Ovarian Tumors - A Tertiary Center Experience. Asian Pacific journal of cancer prevention : APJCP. 2018;19(1):213-8.

22. Moodley M, Bramdev A. Frozen section: Its role in gynaecological oncology. Journal of obstetrics and gynaecology : the journal of the Institute of Obstetrics and Gynaecology. 2005;25(7):629-34.

23. Acikalin A, Torun G, Bagir E, Bayram F, Zeren H, Gulec U, et al. Intraoperative frozen section in ovarian neoplasms; a tertiary center experience. Turk patoloji dergisi. 2014;30(3):184-8.

24. Baker P, Oliva E. A Practical Approach to Intraoperative Consultation in Gynecological Pathology. International Journal of Gynecological Pathology. 2008;27(3):353-65.

25. Gultekin E, Gultekin OE, Cingillioglu B, Sayhan S, Sanci M, Yildirim Y. The value of frozen section evaluation in the management of borderline ovarian tumors. Journal of cancer research and therapeutics. 2011;7(4):416-20.

26. Ratnavelu ND, Brown AP, Mallett S, Scholten RJ, Patel A, Founta C, et al. Intraoperative frozen section analysis for the diagnosis of early stage ovarian cancer in suspicious pelvic masses. The Cochrane database of systematic reviews. 2016;3:Cd010360. 
27. Sukumaran R, Somanathan T, Mathews A, Kattor J, Sambasivan S, Nair RP. Role of frozen section in intraoperative assessment of ovarian masses: a tertiary oncology center experience. Indian journal of surgical oncology. 2014;5(2):99-103.

28. Bige O, Demir A, Saygili U, Gode F, Uslu T, Koyuncuoglu M. Frozen section diagnoses of 578 ovarian tumors made by pathologists with and without expertise on gynecologic pathology. Gynecologic oncology. 2011;123(1):43-6. 